\title{
Manipulation of Powder with Surface Acoustic Wave Actuator to Control Standing and Traveling Modes
}

\author{
Yukako Takizawa, ${ }^{1}$ Yusuke Fukuchi, ${ }^{2}$ Kazuya Hamaguchi, ${ }^{1}$ \\ Satoshi Amaya, ${ }^{3}$ Yuichi Utsumi, ${ }^{4}$ Masahiro Takeo, ${ }^{5}$ \\ Kenji Iimura, ${ }^{5}$ Michitaka Suzuki, ${ }^{6}$ and Tsunemasa Saiki ${ }^{1,7^{*}}$ \\ ${ }^{1}$ Hyogo Prefectural Institute of Technology, 3-1-12, Yukihira-cho, Suma-ku, Kobe 654-0037, Japan \\ ${ }^{2}$ The New Industry Research Organization, 6-1 Minatojima Nakamachi, Chuo-ku, Kobe 650-0046, Japan \\ ${ }^{3}$ Department of Mechanical Engineering, Graduate School of Engineering, The University of Tokyo, \\ 7-3-1, Hongo, Bunkyo-ku, Tokyo 113-8656, Japan \\ ${ }^{4}$ Laboratory of Advanced Science and Technology for Industry, University of Hyogo, \\ 3-1-2, Koto, Kamigori-cho, Ako-gun, Hyogo 678-1205, Japan \\ ${ }^{5}$ Department of Chemical Engineering, Graduate School of Engineering, University of Hyogo, \\ 2167, Shosha, Himeji 671-2280, Japan \\ ${ }^{6}$ Institute for Research Promotion and Collaboration, University of Hyogo, \\ 3F, Jibasan Building, 123, Minamiekimae-cho, Himeji 670-0962, Japan \\ ${ }^{7}$ Department of Electrical Materials and Engineering, Graduate School of Engineering, University of Hyogo, \\ 2167, Shosha, Himeji 671-2280, Japan
}

(Received June 29, 2021; accepted September 6, 2021; online published September 17, 2021)

Keywords: surface acoustic wave (SAW), standing wave, traveling wave, vibration proofing, powder transportation

In this study, we fabricated a new surface acoustic wave (SAW) actuator that can control the SAW mode of a powder-manipulation area between an interdigital transducer (IDT) (for generating the SAW) and an elastic-sheet (chloroprene rubber) pressurizer. In particular, the propagation surface near the ends of the substrate, at which the SAW is reflected, is pressurized with elastic bodies to suppress the reflected wave. We used laser Doppler vibrometry with the fabricated SAW actuator to investigate the change in SAW mode from standing to traveling due to elastic-sheet pressurization. The results of the investigation revealed two key points: (i) the amplitude of the reflected wave could be suppressed by about $90 \%$ by elastic-sheet pressurization and (ii) the SAW could be switched between the standing, traveling, and intermediate modes. We then investigated the behavior of a powder $\left(100-\mu\right.$ m-diameter $\left.\mathrm{SiO}_{2}\right)$ using the SAW actuator. We found that the powder could be dissipated by the SAW in the standing mode and then transported to the IDT side by utilizing the SAW in the traveling mode. It was also shown that a SAW in the intermediate mode between the standing and traveling modes (namely, the amplitude of the reflected wave is about one-third that of the incident wave) can be used to transport the powder to the IDT side while dissipating it.

${ }^{*}$ Corresponding author: e-mail: saiki@hyogo-kg.jp https://doi.org/10.18494/SAM.2021.3489 


\section{Introduction}

In recent years, the miniaturization of chemical analysis systems - used in fields such as medicine, biotechnology, and the environment - has been actively pursued. ${ }^{(1-14)}$ In particular, a "lab-on-a-chip" (LOC) integrates multiple small devices such as pumps, valves, reactors (mixers), and sensors on a single chip, where they are connected by microfluidic channels. ${ }^{(6-14)}$ A LOC has many advantages over conventional analytical techniques, for example, it can measure small amounts of samples and significantly reduce the reaction time and the cost of analysis.

For current LOCs, samples are analyzed in the liquid form; ${ }^{(6-10)}$ however, if dry powders could also be handled, the scope of analysis would be expanded dramatically. Furthermore, dry powders are more chemically stable than liquids, so they are more suitable for storage and easier to handle. Using a dried powder as a sample in a LOC requires a physical operation, namely, the "dry transport" of the powder itself. However, it is technically difficult to simply scale down existing conveyors, blowers, and vibratory feeders in LOCs. Even if it was possible, the resulting LOCs would be very expensive and their operation accuracy with a powder would be lowered significantly.

As devices for transporting such small amounts of powder, ultrasonic and surface acoustic wave (SAW) actuators have been investigated. ${ }^{(15-19)}$ Such actuators convert vibrations produced by the inverse piezoelectric effect into a thrust force (generated by friction) acting on the powder. An ultrasonic actuator is composed of a piezoelectric ring vibrator attached to the end of a transport pipe, and the powder inside the transport pipe is transported by the vibration of the ring. ${ }^{(15-17)}$ In contrast, a SAW actuator is composed of an interdigital transducer (IDT) (consisting of comb-shaped electrodes) placed on a piezoelectric substrate, and by applying a high-frequency voltage to the IDT, a SAW is generated on the substrate and is used to transport powder placed on the substrate. ${ }^{(18,19)}$ Owing to this characteristic, SAW actuators have a much simpler structure than ultrasonic actuators and are thus easier to miniaturize. Accordingly, SAW actuators are the best choice for LOCs, which have a limited area in which to mount components.

However, in a similar manner to ultrasonic actuators, SAW actuators face a problem with their operational mechanism (i.e., principle of operation); that is, individual powder particles cannot be transported efficiently unless they are in contact with the propagation surface. In other words, unlike conveyors, SAW actuators are not suitable for transporting clumps of powder that have accumulated on the substrate. Given this fact, by improving a conventional SAW actuator, which can operate in a single wave mode only, we wondered whether it was possible to change the SAW mode so as to dissipate such powder clumps, thereby increasing the contact area between the individual powder particles and the substrate and making it easier to transport the powder. Accordingly, considering a mechanism to change the SAW mode, we adopted the method of suppressing the reflected wave by pressurizing (with an elastic material) the propagating surface of the SAW (piezoelectric substrate) near its ends, where the SAW is reflected, in a manner that switches it from the standing mode to the traveling mode.

In this study, we first fabricated a novel SAW actuator that can control the standing and traveling modes by pressurizing the SAW propagation plane with an elastic material (chloroprene 
rubber). Then, using the fabricated SAW actuator, we investigated the change in the traveling mode of the SAW (i.e., the decrease in reflected-wave amplitude) from the standing mode due to the elastic-sheet pressure. Finally, we placed a powder on the propagation plane between the SAW-generating IDT and the elastic pressurizer, and we observed the powder behavior in the standing and traveling modes of the SAW and their combination. In that way, we investigated the usefulness of switching SAW modes for powder transport. Hereafter, the results of these observations and investigations are presented and explained.

\section{Change in SAW Mode due to Elastic-sheet Pressure}

\subsection{Measurement method}

A photograph of the prototype SAW actuator used in the experiment is shown in Fig. 1. The SAW actuator was fabricated by patterning a $1-\mu \mathrm{m}$-thick aluminum-film IDT on a $28-\mathrm{mm}$-wide $\times 78$-mm-long piezoelectric substrate $\left(127.8^{\circ}\right.$ rotated Y-cut X-propagating $\mathrm{LiNbO}_{3}$ wafer: 4 inch diameter, $0.5 \mathrm{~mm}$ thickness). The IDT has an $800 \mu \mathrm{m}$ comb pitch, an $8 \mathrm{~mm}$ aperture width, and 10 pairs of comb teeth. The direction perpendicular to the central axis of the IDT is aligned with the orientation-flat direction of the wafer before cutting. This IDT is electrically connected by wires to an SMA connector for external power supply. An elastic chloroprene rubber sheet [CRS-24, Wakisangyo Co., Ltd.; thickness: $2 \mathrm{~mm}$; hardness 60 (durometer type A); size: $10 \times 28$ $\mathrm{mm}$ ] was placed at each end of the piezoelectric substrate (near the points at which the SAW is reflected). Note that the rubber sheets and the piezoelectric substrate were not bonded because the adhesive might act as an absorber.

The experimental system used for observing the displacement of the SAW due to the elasticsheet pressurization by the SAW actuator is shown in Fig. 2. In this system, the RF voltage supplied to the IDT was generated by a function generator (AFG3252, Tektronix Inc.), which generated the $1 \mathrm{kHz}$ burst wave (composed of a 2000 period, $4.2 \mathrm{MHz}$ sine wave) amplified by

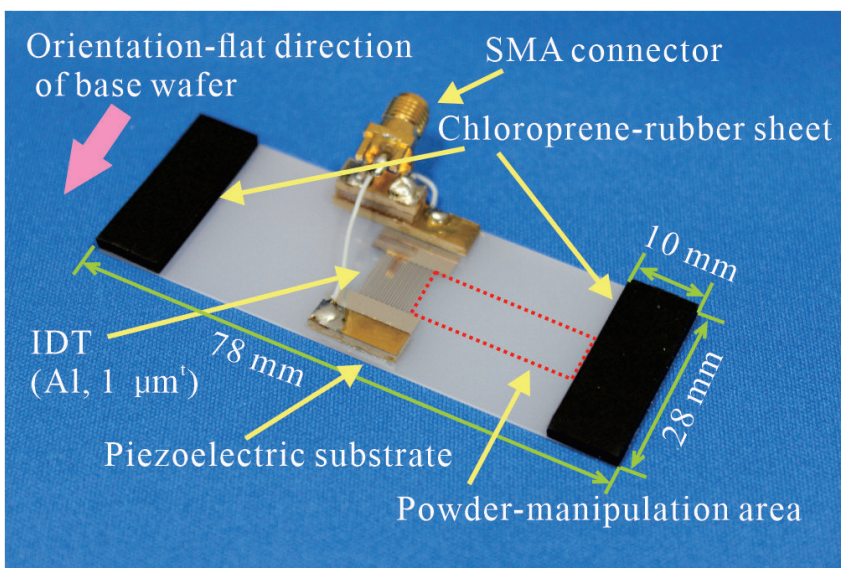

(a)

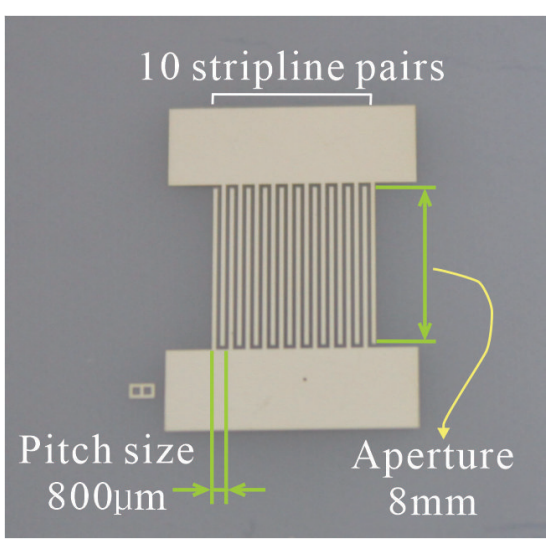

(b)

Fig. 1. (Color online) Photographs of the prototype SAW actuator: (a) overall and (b) magnified views of IDT. 


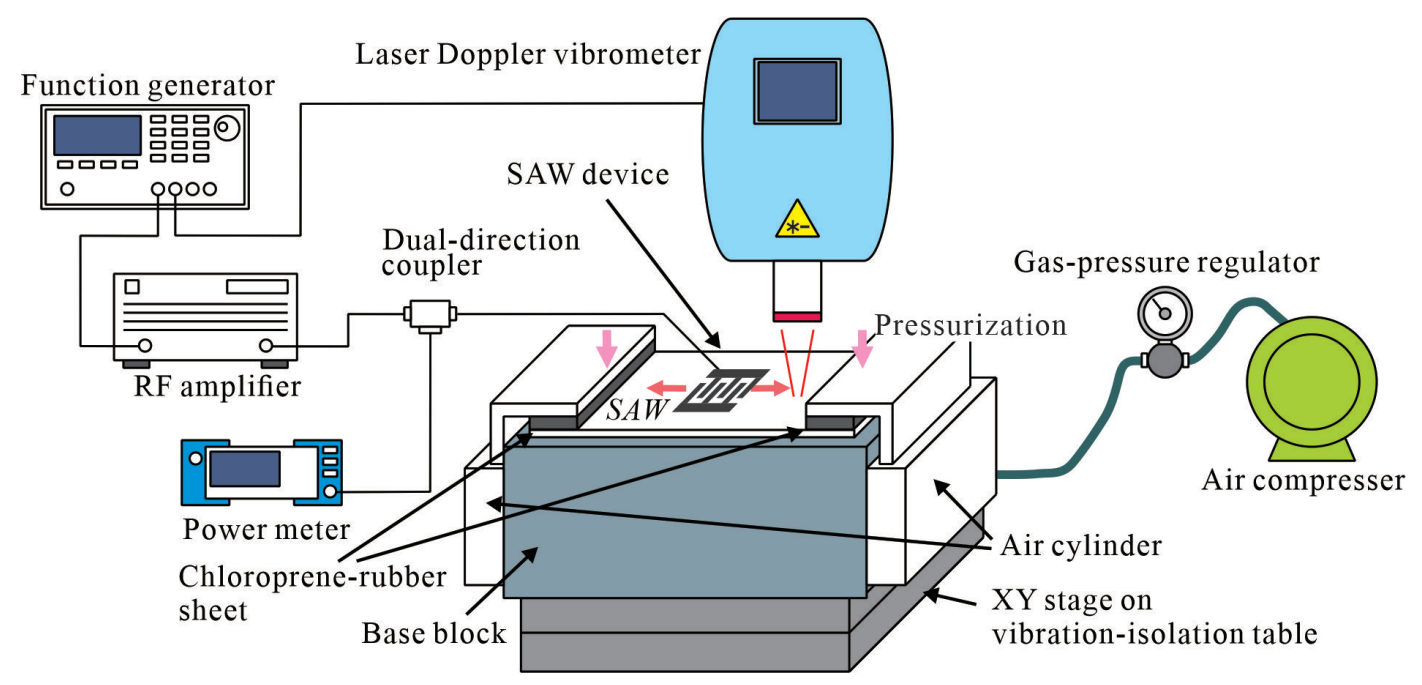

Fig. 2. (Color online) Experimental system for observing SAW displacement due to elastic-sheet pressurization.

an RF amplifier (ALM000110-2840FM, R\&K Co., Ltd.). Note that a burst wave was used to prevent damage to the piezoelectric substrate due to heat. At that time, the set voltage of the function generator was adjusted according to the measured values of incident and reflected power by a power meter (NRP-2, NRP-Z91, Rohde \& Schwarz GmbH \& Co. KG, and DC3001M1, AR Inc.) so that the power supplied to the IDT was $1 \mathrm{~W}$. When this high-frequency voltage was supplied to the IDT, a SAW propagated from the IDT to both ends of the substrate. If the SAW is not attenuated at the elastic sheet, it is reflected at the substrate edge (SAW reflection edge, hereafter) and combined with the incident wave to generate a standing wave. In contrast, if the SAW is attenuated at the elastic sheet and the wave reflected from the edge of the substrate disappears, only the incident wave (i.e., a traveling wave) is visible.

To dampen the SAW, the elastic sheet is pressurized using an air cylinder (MQQTB40, SMC Co.). The air pressure of the air cylinder is controlled by a regulator (IR 1000-01BG, SMC Co., Ltd.), which makes it possible to set the elastic-sheet pressure $(P)$ applied to the surface on which the SAW propagates ("SAW propagation plane" hereafter). In this experimental system, $P$ can be set from 0 to $0.3 \mathrm{MPa}$; however, small $(0.01 \mathrm{MPa}$ ) and large (near $0.3 \mathrm{MPa}$ ) set values were unstable, so the experiments were conducted with $P=0,0.02,0.03,0.05,0.07,0.1$, and $0.2 \mathrm{MPa}$.

To investigate the SAW mode of the powder-manipulation region (between the IDT and the elastic sheets, see Fig. 1) under the various $P$ values via the elastic sheets, we used a laser Doppler vibrometer (MSA-500-TPM2-20-D, Polytech GmbH) to measure the vertical displacement of the SAW on the propagation plane (hereinafter referred to as "SAW displacement"). Note that the SAW propagation plane is set horizontally.

The measurement regions of SAW displacement on the actuator and the coordinates are shown in Fig. 3. As shown in the figure, the $x$-axis is the SAW propagation direction (perpendicular to the stripline of the IDT) and the $y$-axis is the direction perpendicular to the SAW propagation direction. The $x$-coordinate of the origin of the coordinates is the right edge of the IDT and the $y$-coordinate is the center of the IDT aperture. 


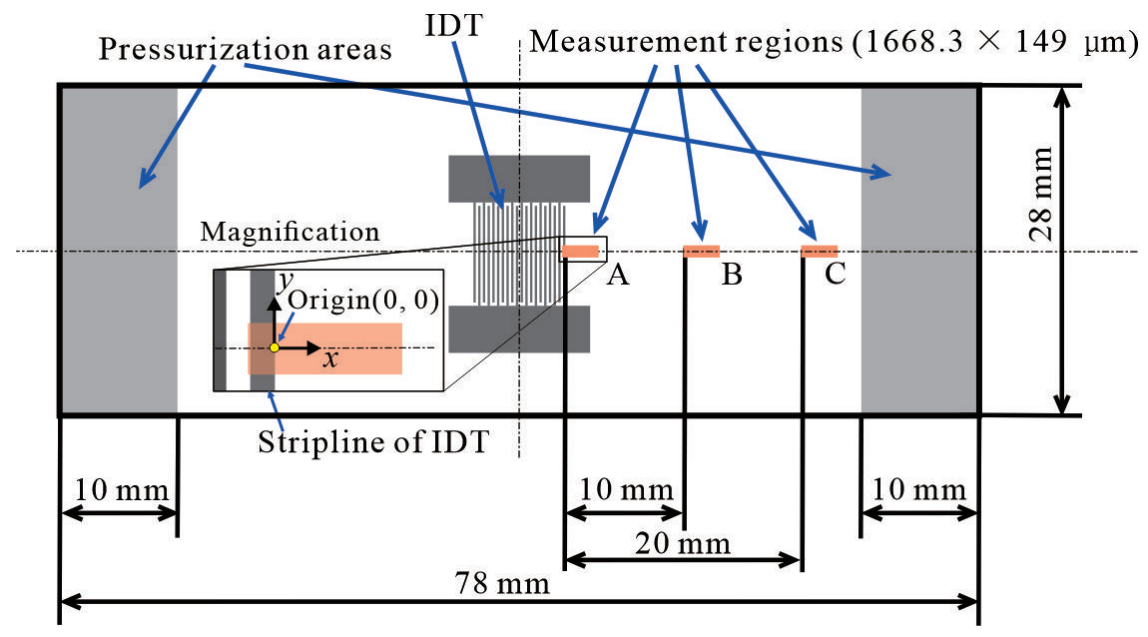

Fig. 3. (Color online) Observation area of SAW displacement and its coordinates.

Actual SAW displacements were measured in the following regions (see Fig. 3): region A, near the IDT ( $x=-223.6$ to $1445.9 \mu \mathrm{m} ; y=-74.5$ to $+74.5 \mu \mathrm{m}$ ); region $\mathrm{B}$, at the center of the SAW propagation plane $10 \mathrm{~mm}$ from the origin in the $x$-direction $(x=9776.4$ to $11445.9 \mu \mathrm{m}$; $y=-74.5$ to $+74.5 \mu \mathrm{m}$ ); and region $\mathrm{C}$, at the SAW propagation edge, $10 \mathrm{~mm}$ further away from region B in the $x$-direction ( $x=19776.4$ to $21445.9 \mu \mathrm{m} ; y=-74.5$ to $+74.5 \mu \mathrm{m})$. SAW displacement was measured at 113 points in the $x$-direction and 11 points in the $y$-direction (a positional resolution of $14.9 \mu \mathrm{m}$ in both the $x$ - and $y$-directions) in all three measurement areas. Since the area that can be measured at one time with the laser vibrometer is limited, SAW displacement in all measurement areas was not measured at once; instead, it was separately measured in each measurement area by moving the laser vibrometer via an XY stage.

\subsection{Measurement results}

In Figs. 4(a) and 4(b), temporal variations of the distribution of SAW displacement in the region near the IDT (region A) when no elastic-sheet pressure was applied to the SAW propagation plane $(P=0 \mathrm{MPa})$ and when the maximum pressure was applied $(P=0.2 \mathrm{MPa})$ are shown, respectively. As can be seen in both figures, the SAW displacement distributions are arranged from top to bottom in time series for every $1 / 8$ th cycle of the voltage applied to the IDT $\{t=(1 / 8) T=1 /(8 f)=1 /(8 \times 4.2 \times 106)[\mathrm{s}]\}$. The displacement scale is shown in the upper right corner of each figure.

First, we focus on the $x$-axis position $(29.8 \mu \mathrm{m})$ at which SAW displacement is maximum at $t$ $=t_{1}$ [top row in Fig. 4(a)] when $P=0 \mathrm{MPa}$. Regarding the temporal variation of SAW displacement at that point (the single-dotted line in the figure), SAW displacement gradually decreases from $t=t_{1}$ to $t=1 / 2 T+t_{1}$ and is almost at its minimum at $t=1 / 2 T+t_{1}$. After that, from $t=1 / 2 T+t_{1}$ to $T+t_{1}$, SAW displacement gradually increases and reaches a value close to the maximum again at $t=T+t_{1}$. This result indicates that the magnitude change in SAW displacement, namely, displacement amplitude, at this point $(x=29.8 \mu \mathrm{m})$ is larger than that in the surrounding areas. 


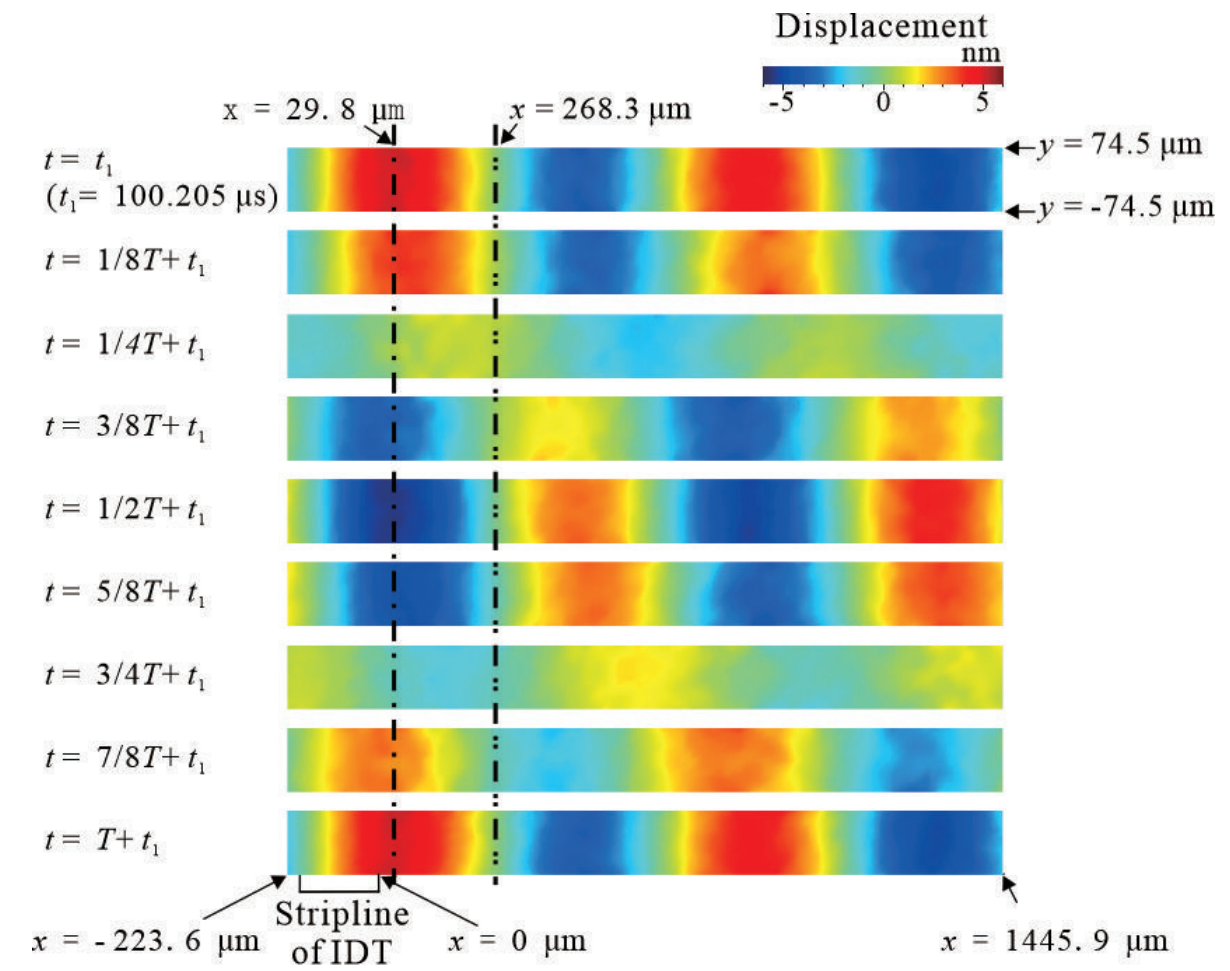

(a)

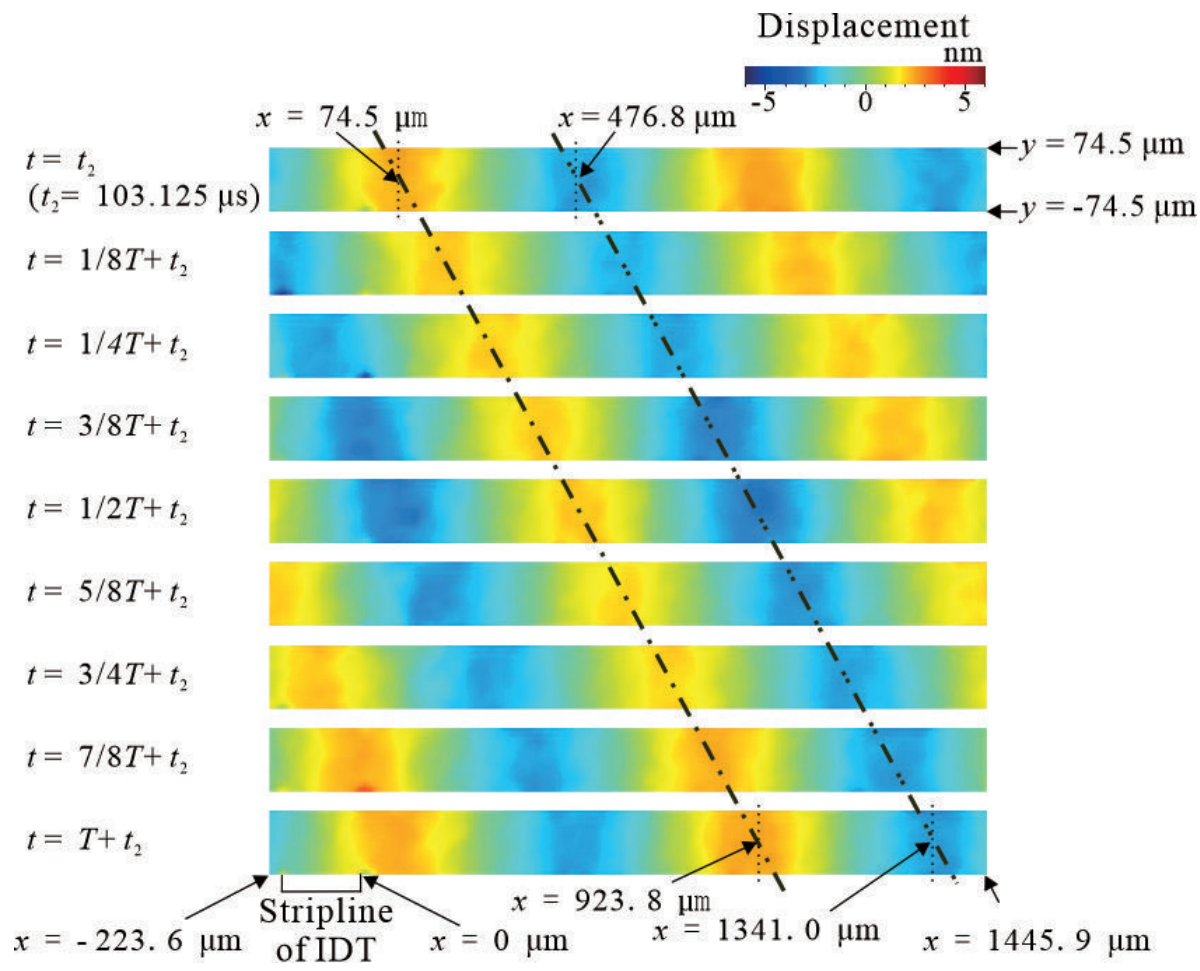

(b)

Fig. 4. (Color online) Temporal changes in distribution of SAW displacement (region A): (a) no pressurization $(P=0 \mathrm{MPa})$ and $(\mathrm{b})$ maximum pressurization $(P=0.2 \mathrm{MPa})$. 
Second, we focus on the $x$-axis position $(268.3 \mu \mathrm{m})$ at which SAW displacement is almost zero at $t=t_{1}$. Similarly, as for the temporal variation at this point (double-dotted line), the displacement amplitude at this point is smaller than that in the surrounding areas. It can be seen from Fig. 4(a) that the points with large and small displacement amplitudes are repeated at regular intervals in the $x$-direction, and the node and antinode characteristics of a standing wave were observed when no elastic-sheet pressurization was applied to the SAW propagation plane. Similar nodes and antinodes characteristic of standing waves were also observed in regions B and $\mathrm{C}$.

Third, we focus on the $x$-axis position $(74.5 \mu \mathrm{m})$ at which SAW displacement is maximum at $t$ $=t_{2}$ [top row of Fig. 4(b)] when $P=0.2 \mathrm{MPa}$. It can be seen that the point at which the maximum displacement is obtained (single-dotted line in the figure) moves to the right (in the direction of increasing $x$ ) with the passage of time. Similarly, the point at which the minimum displacement is obtained (double-dotted line) also moves to the right. The speed of movement of the points at which $x$ has maximum and minimum displacements was calculated as $3.6 \mathrm{~km} / \mathrm{s}$ in both cases. Note that the calculated value is approximately equal to the reported SAW propagation velocity on a $\mathrm{LiNbO}_{3}$ substrate. ${ }^{(20)}$ As described above, when the elastic-sheet pressurization is applied to the propagating plane, the points with the maximum and minimum SAW displacements move at the same speed and in the same direction, which is a characteristic of traveling waves. Similar traveling-wave characteristics were also observed in regions $\mathrm{B}$ and $\mathrm{C}$.

From the above results, we clarified, albeit qualitatively, that the SAW mode can be changed from the standing mode to the traveling mode by using an elastic material to pressurize the SAW propagation plane near the SAW reflection edge of the plane.

\subsection{Quantitative evaluation}

We quantitatively investigated the effects of the elastic-applied pressure $P$ on the SAW propagation plane and the degree of transition of the SAW from the standing mode to the traveling mode on the basis of the time-series data of the SAW displacement distribution. Since total reflection results in a standing wave and no reflection results in a traveling wave, the ratio of the amplitude of the reflected SAW to that of the incident SAW is used as the degree of SAW mode transition. How this ratio is obtained is explained in detail as follows.

Time evolutions of SAW displacement at measurement point $x=29.8 \mu \mathrm{m}, y=0 \mu \mathrm{m}$ with no elastic body pressure $(P=0 \mathrm{MPa})$ and with the maximum pressure $(P=0.2 \mathrm{MPa})$ are shown in Figs. 5(a) and 5(b), respectively. As mentioned in the previous section, this measurement point corresponds to the antinode of the standing wave when no pressure is applied, and $t=0 \mu$ s is the time when the power supply is switched on, i.e., when the high-frequency voltage is applied to the IDT. For reference, the time evolution of the high-frequency voltage (a $4.2 \mathrm{MHz}$ sine wave with 2000 periods) applied to the IDT is shown in Fig. 5(c).

The time evolution of SAW displacement at $P=0 \mathrm{MPa}$ in Fig. 5(a) shows that the amplitude of SAW displacement is about 2 to $5 \mathrm{~nm}$ at about the time the RF voltage is applied to the IDT, and this result confirms that a SAW is generated. Note that the amplitude of SAW displacement of about $2 \mathrm{~nm}$ before the application of the voltage is that of a dark signal generated by the laser 


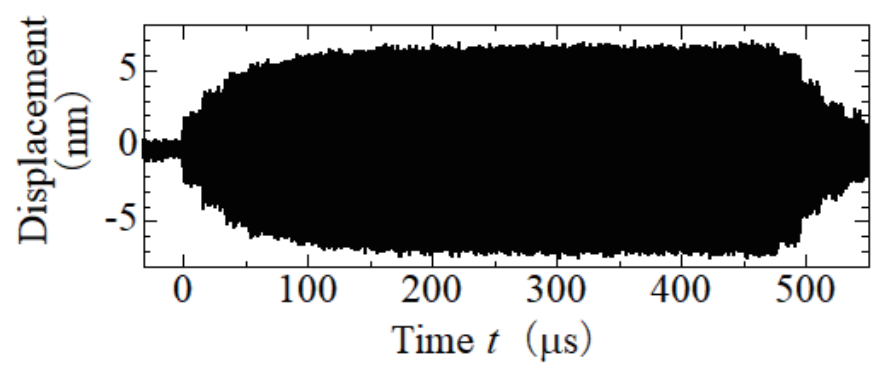

(a)

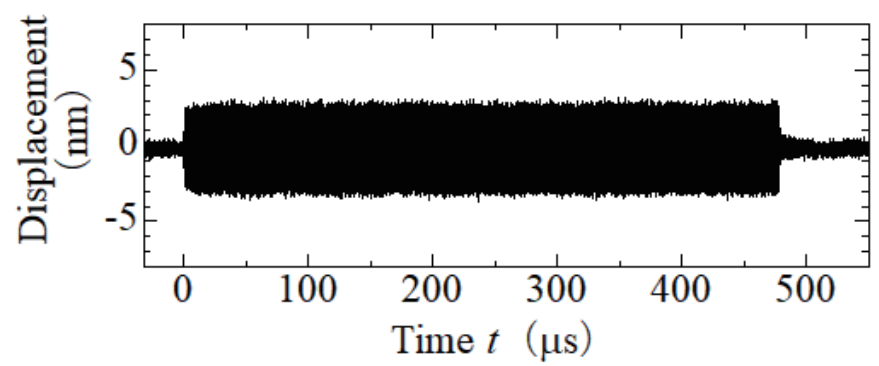

(b)

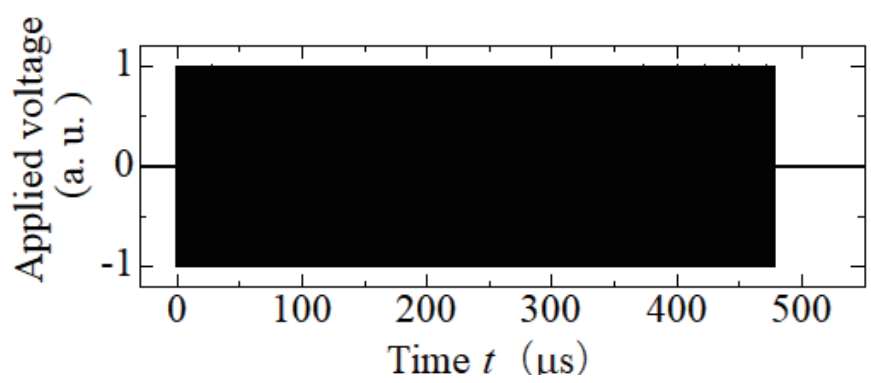

(c)

Fig. 5. Time evolutions of SAW displacement at one measurement point ( $x=29.8 \mu \mathrm{m}, y=0 \mu \mathrm{m})$ : (a) without pressure $(P=0 \mathrm{MPa}),(\mathrm{b})$ at maximum pressure $(P=0.2 \mathrm{MPa})$, and (c) (reference) RF voltage applied to the IDT.

vibrometer used in the experiment, and it does not mean that the SAW propagation plane is actually fluctuating. From the time when the tip of the SAW generated by the IDT is reflected back $(t=16 \mu \mathrm{s})$, the reflected and traveling waves start to combine, and the SAW displacement amplitude gradually increases. After that, the SAW displacement amplitude reaches a maximum of about $14 \mathrm{~nm}$ at around $150 \mu \mathrm{s}$, and it remains at that level until after $450 \mu \mathrm{s}$, when voltage is no longer applied to the IDT. In contrast, the time evolution of SAW displacement at $P=0.2 \mathrm{MPa}$ in Fig. 5(b) shows that the amplitude of SAW displacement is constant at about half the maximum amplitude in the unpressurized state while the high-frequency voltage is applied to the IDT, which indicates that the SAW is not reflected back. On the basis of the time evolutions of SAW displacement shown in Figs. 5(a) and 5(b), the absolute value of SAW displacement $d(t, x, y)$ at each measurement point $(x, y)$ is integrated from time $t_{a}$ to $t_{b}$ to obtain its time-averaged $D_{\text {ave }}(x, y)$ as

$$
D_{\text {ave }}(x, y)=\frac{1}{t_{b}-t_{a}} \int_{t_{a}}^{t_{b}}|d(t, x, y)| d t .
$$


In Eq. (1), $t_{a}$ and $t_{b}$ were set to 150 and $450 \mu \mathrm{s}$, respectively, according to the time when the standing wave was stably generated [see Fig. 5(a)], and only $D_{\text {ave }}(x, y)$ at $y=0$ was calculated as a representative because SAW displacement is considerably larger in the $x$-direction than in the $y$-direction (see Fig. 4). Note that $D_{a v e}(x, 0)$ obtained from Eq. (1) is a function of the absolute value of a sine wave $(|\alpha \sin (\beta \mathrm{x}+\gamma)|)$ if the SAW is an ideal standing wave, and it is a uniform function $(\alpha / 2)$ if the SAW is an ideal traveling wave. Here, $\alpha, \beta$, and $\gamma$ are constants.

$D_{\text {ave }}(x, 0)$ values in regions $\mathrm{A}, \mathrm{B}$, and $\mathrm{C}$ are plotted in Figs. 6(a)-6(c), respectively. These plots show that in all regions, as the elastic pressure $P$ applied to the SAW propagation plane increases, $D_{\text {ave }}(x, 0)$ approaches a uniform function from a sinusoidal function with an absolute value; in other words, the SAW shifts from the standing mode to the traveling mode. To quantitatively determine the degree of transition of the SAW mode, the range of $D_{\text {ave }}(x, 0)$ (maximum value - minimum value) for each $P$ was investigated, and the $D_{\text {ave }}(x, 0)$ range without pressure $(P=0 \mathrm{MPa})$ was used as a reference for calculating the ratio of the $D_{\text {ave }}(x, 0)$ range at each $P$. These $D_{\text {ave }}$ values for each region are summarized in Fig. 7. Theoretically, the amplitude of the reflected wave when the elastic sheet is not pressurized (standing-wave case) is equal to

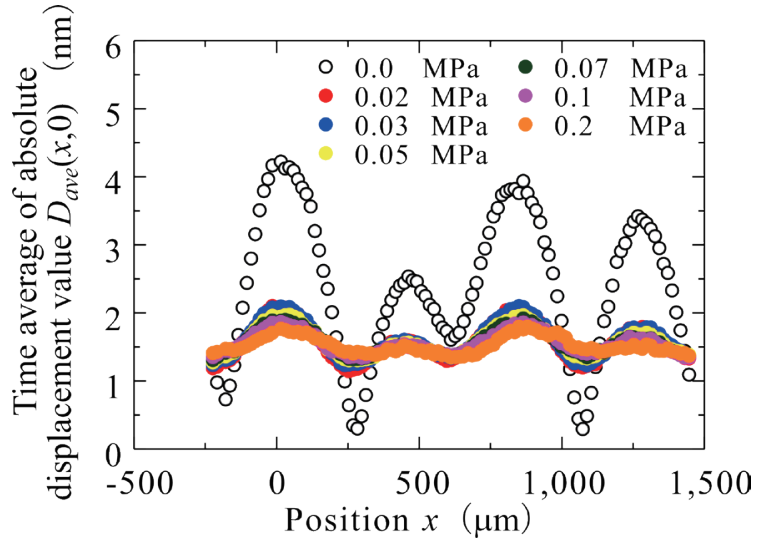

(a)

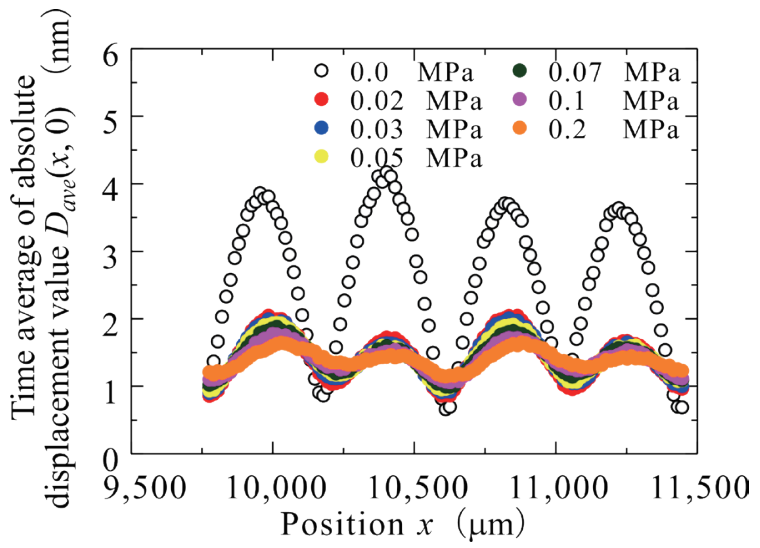

(b)

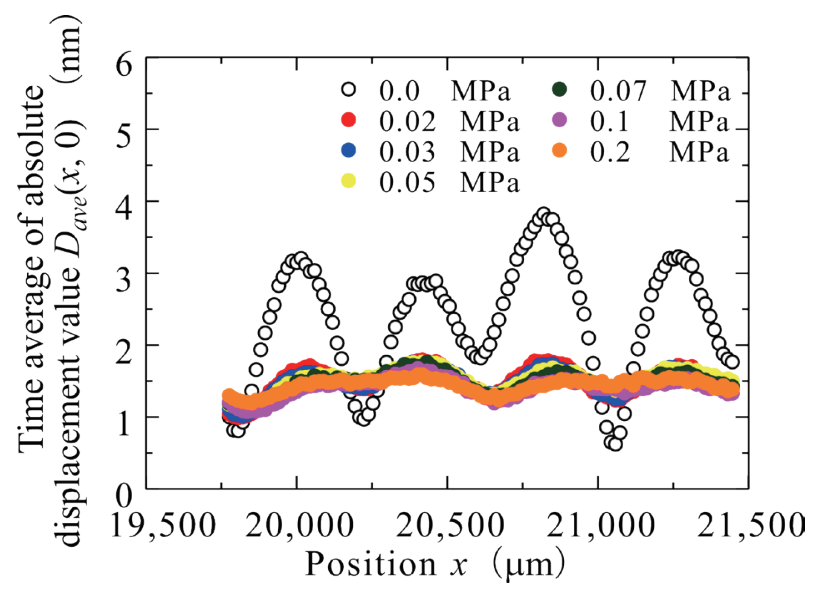

(c)

Fig. 6. (Color online) Time-averaged absolute values of SAW displacements at each position $x$ : (a) region A, (b) region $\mathrm{B}$, and $(\mathrm{c})$ region $\mathrm{C}$. 


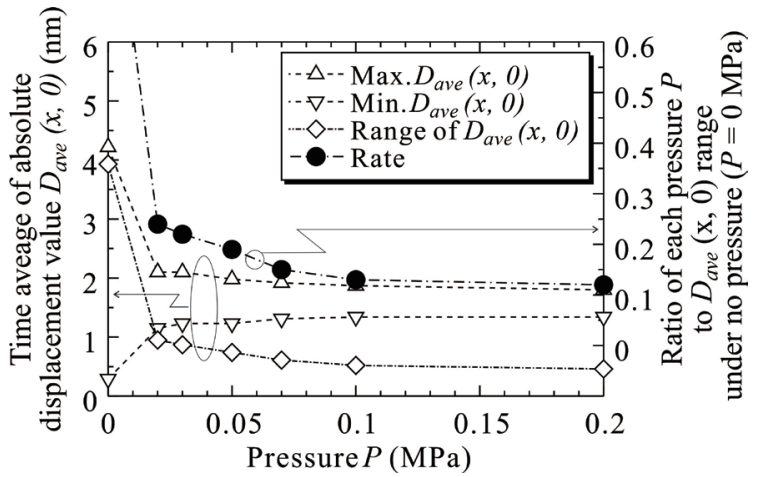

(a)

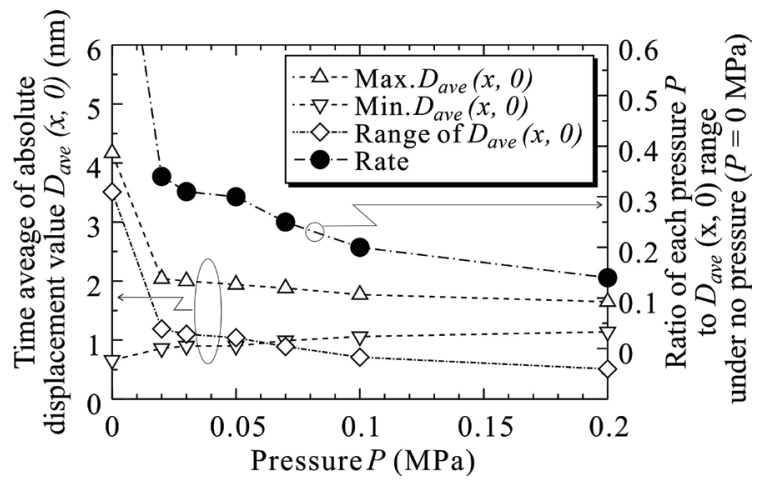

(b)

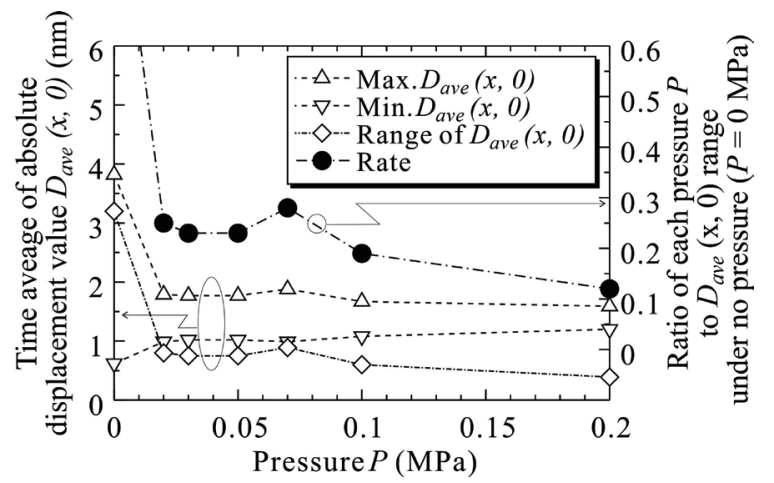

(c)

Fig. 7. Ratio of each pressure $P$ to $D_{\text {ave }}(x, 0)$ range under no pressure $(P=0 \mathrm{MPa})$ : (a) region $\mathrm{A}$, (b) region $\mathrm{B}$, and (c) region $\mathrm{C}$.

that of the incident wave; accordingly, the ratio of the obtained range of $D_{\text {ave }}(x, 0)$ represents the ratio of the reflected-wave amplitude to the incident-wave amplitude under elastic-sheet pressurization $P$. It is therefore concluded that the amplitude of the reflected wave can be reduced by about 70 to $90 \%$ of the incident wave by increasing $P$ from 0.02 to $0.2 \mathrm{MPa}$, although some differences exist in each region. In other words, by applying $P$ of $0.2 \mathrm{MPa}$ with an elastic sheet to the propagation plane near the SAW reflection edge, the reflected wave became almost negligible compared with the incident wave. It was thus quantitatively clarified that the SAW in the powder-manipulation area is in the traveling mode.

\section{Powder Behavior Due to Standing and Traveling Waves}

As described in the previous section, when applying pressure to the SAW propagation plane near the SAW reflection edge via an elastic sheet, the amplitude of the reflected wave decreases with increasing elastic-sheet pressure. As described in this section, a powder was placed between the IDT and the elastic-sheet pressurizer (the powder-manipulation region), and the powder behavior in the standing and traveling modes of the SAW and their combination was observed, and the feasibility of transporting powder by switching the SAW mode is discussed. In the 
experiment, glass beads (ASGB-180, AS ONE Co.; specific gravity: 2.5; particle size: 0.090$0.106 \mathrm{~mm}$ ) were used as the test powder, and this powder (a $7 \mathrm{~mm}^{3}$ clump of glass beads) was placed $15 \mathrm{~mm}$ in front of the IDT on the substrate of the SAW actuator by using a self-made cylindrical container ( $3 \mathrm{~mm}$ diameter and $1 \mathrm{~mm}$ depth). The behavior of the powder subjected to the SAW was then observed with a high-definition camera (HDR-CX430V, SONY Co., Ltd.). In this experiment, the IDT was supplied with the same burst voltage (power: $1 \mathrm{~W}$ ) as that when SAW displacement was measured. The elastic pressure on the SAW propagation plane was set to $P=0,0.2$, or $0.02 \mathrm{MPa}$, and the behavior of the powder subjected to the SAW in the standing, traveling, and intermediate modes was investigated. Moreover, the powder behavior was also investigated when the SAW shifted from the standing mode $(P=0 \mathrm{MPa})$ to the traveling mode $(P=0.2 \mathrm{MPa}) 30 \mathrm{~s}$ after the power supply was switched on. The investigations were conducted at atmospheric pressure at a temperature of $24{ }^{\circ} \mathrm{C}$ and a humidity of $54 \%$.

Time-series photographs of the powder behavior under the above conditions are shown in Fig. 8. First, the powder behavior under the standing mode is focused on [Fig. 8(a)]. From the start of the power supply ( $t=0 \mathrm{~s}$ ) to $10 \mathrm{~s}$ later, the powder clump spreads out, i.e., the powder clump dissipates. During this time, the powder moves by about $5 \mathrm{~mm}$ to the IDT side. However, the powder does not move further to the IDT side after $10 \mathrm{~s}$; instead, it spreads out further in other directions. As the powder spreads out, longitudinal stripes appear, with finer and coarser
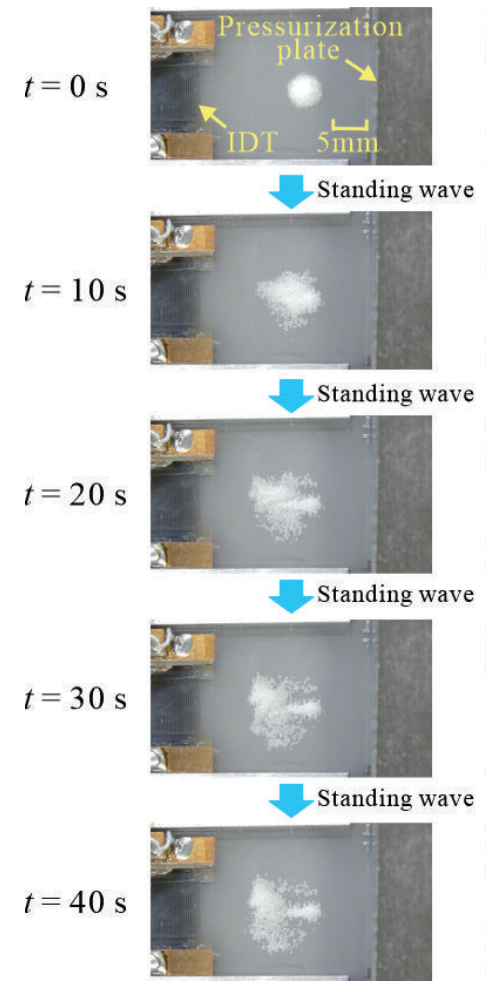

(a)

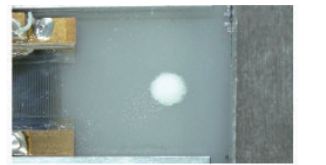

Traveling wave
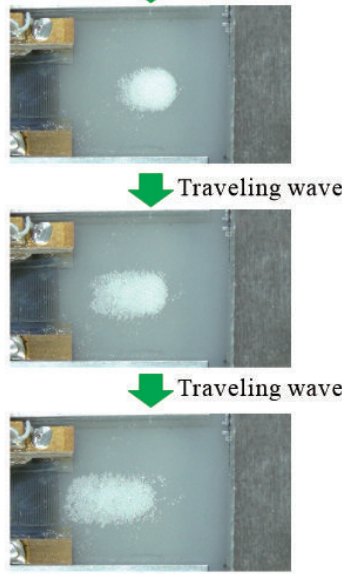

Traveling wave

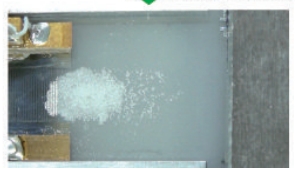

(b)

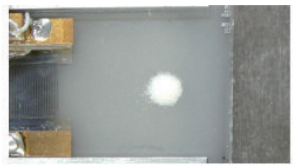

Mixed wave

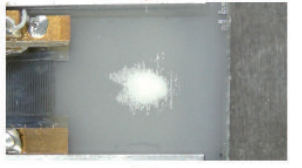

Mixed wave
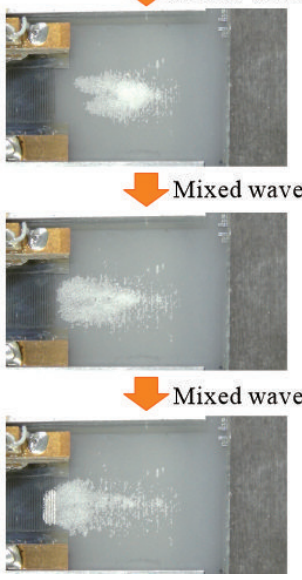

(c)

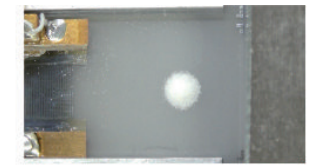

Standing wave

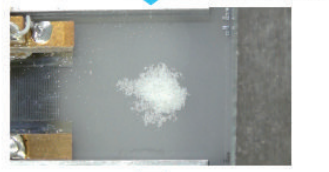

Standing wave
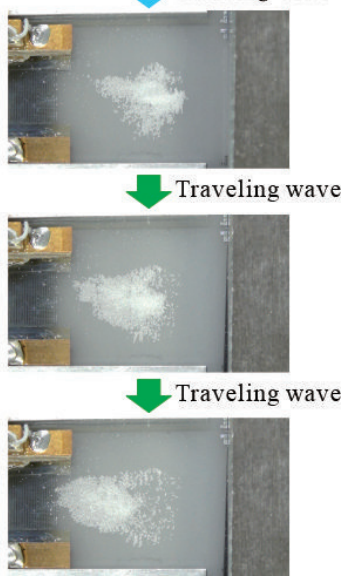

(d)

Fig. 8. (Color online) Time-series photographs of powder behavior under each condition: (a) standing mode $(P=0 \mathrm{MPa})$, (b) traveling mode $(P=0.2 \mathrm{MPa}),(\mathrm{c})$ intermediate mode $(P=0.02 \mathrm{MPa})$, and $(\mathrm{d})$ standing + traveling mode. 
particles respectively corresponding to the nodes and antinodes of the standing wave. In other words, the SAW in the standing mode can collapse $\mathrm{SiO}_{2}$ powder clumps, but it cannot transport them. In contrast, when the powder behavior in the traveling mode is focused on [Fig. 8(b)], it becomes clear that the behavior in the traveling mode is different from that in the standing mode. In particular, almost no vertical stripes appear owing to variations in the density of the powder. Also, although the powder spreads out to a certain extent, it is efficiently transported to the IDT side.

The powder behavior in the intermediate mode [Fig. 8(c)] is between those in the standing mode [Fig. 8(a)] and traveling mode [Fig. 8(b)]. Looking at the powder behaviors in Figs. 8(a)8 (c) again from the viewpoint of powder transport, we see that not only the traveling-mode SAW but also the intermediate-mode SAW has higher transport efficiency than the standing-mode SAW.

Next, we focused on the powder behavior due to the SAW when the mode is changed from standing to traveling [Fig. 8(d)]. We clearly see that, as originally expected, the powder clump collapsed and dissipated when subjected to the SAW in the standing mode and was then transported by the SAW in the traveling mode. However, it is also clear that although the powder clump can be broken down and then transported, the transport efficiency was higher than that in the standing mode but not as high as that in the traveling mode. We think that this result can be explained by a slight delay in the timing of switching the modes.

\section{Concluding Remarks}

In this study, to investigate the possibility of improving the efficiency of powder transport by a SAW, we fabricated a novel SAW actuator that can control the standing and traveling modes by applying elastic pressure to the SAW propagation plane near the SAW reflection edge. The SAW actuator was then used to investigate the change in SAW mode from standing to traveling with elastic-sheet pressurization, i.e., the ratio of the reflected-wave amplitude to the incident-wave amplitude. We found that (i) the reflected-wave amplitude could be suppressed by about $90 \%$ by pressurizing the SAW propagation plane with an elastic sheet and that (ii) the SAW mode could be freely changed from standing to traveling.

The behavior of a powder $\left(\mathrm{SiO}_{2}\right.$ with a particle diameter of $\left.100 \mu \mathrm{m}\right)$ due to the $\mathrm{SAW}$ in the standing and traveling modes was investigated using the fabricated SAW actuator. It was found that the powder could be scattered but not transported in one direction by using the SAW in the standing mode; however, it could be transported by using the SAW in the traveling mode. Furthermore, a SAW (namely, a reflected wave with about one-third of the amplitude of the incident wave) in the intermediate mode between the standing and traveling modes could be used to transport the powder, although some of the powder dissipated. The temporal switching of the SAW mode from standing to traveling allowed the powder clump to be dispersed and then transported. In the future, by adjusting parameters such as the ratio of reflected waves to incident waves and the timing of switching from the standing mode to the traveling mode, we plan to optimize powder transport by a SAW in the intermediate mode and by switching the SAW mode from standing to traveling. 


\section{Acknowledgments}

This research was supported by Grant-in-Aid for Scientific Research (C) No. 16K06170 from the Japan Society for the Promotion of Science (JSPS).

\section{References}

1 A. Manz, N. Graber, and H. M. Widmer: Sens. Actuators, B 1 (1990) 244. https://doi.org/10.1016/09254005(90)80209-I

2 D. R. Reyes, D. Iossifidis, P. A. Auroux, and A. Manz: Anal. Chem. 74 (2002) 2623. https://doi.org/10.1021/ ac0202435

3 P. A. Auroux, D. Iossifidis, D. R. Reyes, and A. Manz: Anal. Chem. 74 (2002) 2637. https://pubs.acs.org/ doi/10.1021/ac020239t

4 T. Vilkner, D. Janasek, and A. Manz: Anal. Chem. 76 (2004) 3373. https://pubs.acs.org/doi/10.1021/ac040063q

5 M. L. Kovarik, D. M. Ornoff, A. T. Melvin, N. C. Dobes, Y. Wang, A. J. Dickinson, P. C. Gach, P. K. Shah, and N. L. Allbritton: Anal. Chem. 85 (2013) 451. https://doi.org/10.1021/ac3031543

6 A. W. Browne, L. Ramasamy, T. P. Cripe, and C. H. Ahn: Lab Chip 11 (2011) 2440. https://doi.10.1039/ c1lc20144a

7 T. U. Vinitha, G. Sthitodhi, M. Alexander, N. Thinh, and H. A. Chong: Lab Chip 20 (2020) 1961. https://doi. org/10.1039/D0LC00071J

8 N. Pamme, R. Koyama, and A. Manz: Lab Chip 3 (2003) 187. https://doi.org/10.1039/B300876B

9 K. L. Saar, Y. Zhang, T. Müller, C. P. Kumar, S. Devenish, A. Lynn, U. Łapińska, X. Yang, S. Linsed, and T. P. J. Knowles: Lab Chip 18 (2018) 162. https://doi.org/10.1039/c7lc00797c.

10 B. C. Dhar and N. Y. Lee: Bio Chip J. 12 (2018) 173. https://doi.org/10.1007/s13206-018-2301-5

11 Y. Kikutani, T. Horiuchi, K. Uchiyama, H. Hisamoto. M. Tokeshi, and T. Kitamori: Lab Chip 2 (2002) 188. https://doi.org/10.1039/B208382P

12 S. Balbach and C. Korn: Int. J. Pharm. 275 (2004) 1. https://doi.org/10.1016/j.ijpharm.2004.01.034

13 P. S. Dittrich and P. Schwille: Anal. Chem. 75 (2003) 5767. https://doi.org/10.1021/ac034568c

14 M. Shirai, K. Arikawa, K. Taniguchi, M. Tanabe, and T. Sakai: Sci. Rep. 23 (2016) 1. https://doi.org/10.1038/ $\underline{\text { srep36014 }}$

15 M. Mracek and J. Wallaschek: Mater. Chem. Phys. 90 (2005) 378. https://doi.org/10.1016/j. matchemphys.2004.09.048

16 N. Kanbe, Y. Tomikawa, and T. Takano: Jpn. J. Appl. Phys. 32 (1993) 2405. https://doi.org/10.1143/JJAP.32.2405

17 U. Kuhne and U. Fristsching: Powder Tech. 155 (2005) 117. https://doi.org/10.1016/j.powtec.2005.05.050

18 T. Saiki, A. Tsubosaka, A. Yamaguchi, M. Suzuki, and Y. Utsumi: Adv. Powder Tech. 28 (2017) 491. https://doi. org/10.1016/j.apt.2016.11.006

19 T. Saiki, Y. Takizawa, T. Kaneyoshi, K. Iimura, M. Suzuki, A. Yamaguchi, and Y. Utsumi: Sens. Mater (in press).

20 G. Kovacs, M. Anhorn, H. E. Engan, G. Visintini, and C. C. W. Ruppel: Ultrason. Symp. Proc. (1990) 435. https://doi.org/10.1109/ULTSYM.1990.171403 